\title{
Blau syndrome
}

INSERM

\section{Source}

INSERM. (1999). Orphanet: an online rare disease and orphan drug data base. Blau syndrome. ORPHA:90340

Blau syndrome (BS) is a rare systemic inflammatory disease characterized by early onset granulomatous arthritis, uveitis and skin rash. BS now refers to both the familial and sporadic (formerly early-onset sarcoidosis) form of the same disease. The proposed term pediatric granulomatous arthritis is currently questioned since it fails to represent the systemic nature of the disease. 